УДК 517.95

\title{
On an Analogue of the Riemann-Hilbert Problem for a Non-linear Perturbation of the Cauchy-Riemann Operator
}

\author{
Yulia L. Cherepanova* \\ Alexander A. Shlapunov ${ }^{\dagger}$ \\ Institute of Mathematics and Computer Science \\ Siberian Federal University \\ Svobodny, 79, Krasnoyarsk, 660041
}

Russia

Received 16.08.2016, received in revised form 06.09.2016, accepted 15.10.2016

\begin{abstract}
We consider a non-linear perturbation of a famous Riemann-Hilbert problem on the recovering of a holomorphic function in a domain via its real part on the boundary. We get an information on the local structure of the solutions and give sufficient conditions for their real analyticity. A simple instructive example is considered.
\end{abstract}

Keywords: the Cauchy-Riemann operator, non-linear Riemann-Hilbert problem. DOI: $10.17516 / 1997-1397-2016-9-4-427-431$.

Various non-linear generalizations of the famous Riemann-Hilbert problem on the recovering of a holomorphic function $f$ in a domain $D$ on the complex plane $\mathbb{C}$ via a linear combination of its imaginary $\operatorname{Im}(f)$ and real $\operatorname{Re}(f)$ parts on the boundary $\partial D$ of $D$ (see [1]) were discussed in many aspects, see, for instance, [2-6]. The aim of this short note is the investigation of a non-linear generalization of the Riemann-Hilbert problem from the viewpoint of the differential equations.

More precisely, for the closure $\bar{O}$ of an open set $O \subset \mathbb{C}$ we denote by $\mathcal{C}^{s, \lambda}(\bar{O})$ the space of functions satisfying the Hölder condition with a power $0<\lambda<1$ together with all its derivatives up to order $s \in \mathbb{Z}_{+}$on $\bar{O}$. We also denote by $\bar{\partial}=\frac{1}{2}\left(\frac{\partial}{\partial x}+\sqrt{-1} \frac{\partial}{\partial y}\right)$ the Cauchy-Riemann operator on the plane $\mathbb{C} \cong \mathbb{R}^{2}$ with the coordinates $z=x+\sqrt{-1} y$, and consider the following problem.

Problem 1. Given functions $a(z) \in \mathcal{C}^{0, \lambda}(\bar{D}), u_{0} \in \mathcal{C}^{1, \lambda}(\partial D)$ and a given meromorphic function $F(f)$ over $\mathbb{C}$, find a function $f \in \mathcal{C}^{1, \lambda}(\bar{D} \backslash S)$ such that

$$
\left\{\begin{array}{lll}
\bar{\partial} f=a(z) F(f) & \text { in } & D \backslash S, \\
B(f)=u_{0} & \text { on } & \partial D,
\end{array}\right.
$$

where $S$ is a closed set containing poles of the function $F$ and $B$ is a suitable non-linear operator.

Of course, in the present form the problem is too immense. In the most simple cases, $F(f)=0$ or $F(f)=f$, and $B(f)=\operatorname{Re}(f)$, Problem 1 becomes linear one. In these cases it can be easily reduced to the Riemann-Hilbert problem (see, for instance, [1]). In this note we consider a natural class for the function $F$, such that a helpful information on the local structure of the

\footnotetext{
*yuliyacherepanova@mail.ru

†ashlapunov@sfu-kras.ru

(c) Siberian Federal University. All rights reserved
} 
non-linear Problem 1. We also consider a simple but instructive example where this information appears to be global and allows to construct formulas for solutions of the problem.

Let $\mathcal{M}(O)$ stand for the class of meromorphic functions on an open set $O \subset \mathbb{C}$. Let also $N(F)$ stand for the set of zeros of the function $F$.

Lemma 1. Let $D$ be a domain with the boundary $\partial D$ class $C^{2}, a \in C^{0, \lambda}(\bar{D}), F(w) \in \mathcal{M}(\mathbb{C})$, the function $\frac{1}{F(w)}$ admit a primitive $G(w) \in \mathbb{C} \backslash N(F)$, and $h(z)$ be a function holomorphic in a neighbourhood of a point $z_{0} \in D$. If $G(w)$ is an invertible map in a neighbourhood of the point $h\left(z_{0}\right) \in \mathbb{C}$ then the function

$$
f(z)=G^{-1}\left(A(z)-A\left(z_{0}\right)+h(z)\right)
$$

is a solution to the equation

$$
(\bar{\partial} f)(z)=a(z) F(f(z))
$$

in a neighbourhood of the point $z_{0}$.

Proof. First of all we invoke the Potential Theory (see, for instance, [7]). It implies that for any complex function $a \in C^{0, \lambda}(\bar{D})$ there is a complex potential

$$
A(z)=\frac{1}{2 \pi \sqrt{-1}} \int_{D} \frac{a(\zeta)}{\zeta-z} d \bar{\zeta} \wedge d \zeta
$$

(here $\bar{\zeta}$ is the complex adjoint to the complex variable $\zeta$ ) belonging to the Hölder space $C^{1, \lambda}(\bar{D})$ and satisfying $\bar{\partial} A(z)=a(z)$ in $D$.

Let $h$ be holomorphic in a neighbourhood $U\left(z_{0}\right)$ of the point $z_{0} \in D$ and $G(w)$ be an invertible holomorphic map in a neighbourhood $V\left(h\left(z_{0}\right)\right)$ of the point $h\left(z_{0}\right) \in \mathbb{C}$. As the potential $A$ is continuous, there is a neighbourhood $W\left(z_{0}\right)$ of the point $z_{0}$ such that $A(z)-A\left(z_{0}\right)+h(z) \in$ $V\left(h\left(z_{0}\right)\right)$ for all $z \in W\left(z_{0}\right)$. Since the map $G(w)$ admits the inverse map $G^{-1}$ on the set $V\left(h\left(z_{0}\right)\right)$ then the function

$$
f(z)=G^{-1}\left(A(z)-A\left(z_{0}\right)+h(z)\right)
$$

is defined on $W\left(z_{0}\right)$. In particular,

$$
G(f(z))=A(z)-A\left(z_{0}\right)+h(z)
$$

for all $z \in W\left(z_{0}\right)$. Applying the Cauchy-Riemann operator $\bar{\partial}$ to the function $G(f(z))$ we obtain $\bar{\partial} G(f(z))=a(z)$ for all $z \in W\left(z_{0}\right)$. On the other hand, by the formula for the differentiation of the composition of maps, we get

$$
\bar{\partial} G(f(z))=G^{\prime}(f(z)) \bar{\partial} f(z)
$$

for all $z \in W\left(z_{0}\right)$. As $G$ is a primitive for the function $1 / F(w)$ we see that

$$
G^{\prime}(f(z)) \bar{\partial} f(z)=a(z)
$$

for all $z \in W\left(z_{0}\right)$. Hence $\bar{\partial} f(z)=F(f(z)) a(z)$ in a neighbourhood of $z_{0}$.

Lemma 2. Let $D$ be a domain with the boundary $\partial D$ class $\mathcal{C}^{2}, \partial D, a \in C^{0, \lambda}(\bar{D}), F(w) \in \mathcal{M}(\mathbb{C})$, the function $\frac{1}{F(w)}$ admits a primitive $G(w) \in \mathbb{C} \backslash N(F)$ and the map $G(w)$ is invertible in a neighbourhood of the point $f\left(z_{0}\right)$ for some point $z_{0} \in D$. If $f \in C^{1, \lambda}(\bar{D})$ and

$$
\bar{\partial} f(z)=a(z) F(f(z))
$$


then there is a function $h_{0}$, holomorphic in a neighbourhood of the point $z_{0}$, such that

$$
f(z)=G^{-1}\left(A(z)-A\left(z_{0}\right)+h_{0}(z)\right) .
$$

Proof. We set

$$
h_{0}(z)=G(f(z))-A(z)+A\left(z_{0}\right)
$$

This function is well defined in the neighbourhood of the point $z_{0}$ where the function $G(f(z))$ is defined. By the definition $h_{0}$ is $\mathbb{R}$-differentiable the neighbourhood. Moreover, as $G$ is a primitive of the function $\frac{1}{F(w)}$, then again using the formula for the differentiation of the composition of maps, we obtain

$$
\bar{\partial} h_{0}(z)=0 .
$$

Hence the function $h_{0}(z)$ is holomorphic in a neighbourhood of the point $z_{0} \in D$.

These two technical lemmas lead to the following useful theorem.

Theorem 1. Let $a(z) \in C^{0, \lambda}(\bar{D})$ be real analytic in $D, F(w) \in \mathcal{M}(\mathbb{C})$ and the function $\frac{1}{F(w)}$ admit a primitive $G(w) \in \mathbb{C} \backslash N(F)$. If $f \in \mathcal{C}^{1, \lambda}(\bar{D})$ and $\bar{\partial} f(z)=a(z) F(f(z))$ in $D$ and the map $G(w)$ is invertible in a neighbourhood of the point $f\left(z_{0}\right)$ with $z_{0} \in D$ then $f$ is real analytic in this point.

Proof. Using Lemma 2 for the point $z_{0}$ described in the theorem, we conclude that there are a neighbourhood and a holomorhic function $h_{0}$ in this neighbourhood such that

$$
f(z)=G^{-1}\left(A(z)-A\left(z_{0}\right)+h_{0}(z)\right) .
$$

Besides, if the function $a(z) \in \mathcal{C}^{0, \lambda}(\bar{D})$ is real analytic, in $D$ then, by Petrovskii Theorem on realanalyticity of solutions to elliptic equations, the potential $A(z)$ is real analytic function in $D$, too. As any holomorphic function is real analytic we conclude that the function $A(z)-A\left(z_{0}\right)+h(z)$ is real analytic in a neighbourhood of the point $z_{0}$.

Since $F(w) \in \mathcal{M}(\mathbb{C})$ we see that $\frac{1}{F(w)}$ is holomorphic everywhere accept zeros of the function $F$. As $G(w)$ admits the inverse map in a neighbourhood of the point $f\left(z_{0}\right) \in \mathbb{C}$, the Inverse Map Theorem implies that $G^{-1}(w)$ is holomorphic function in a neighbourhood of the point $f\left(z_{0}\right)$. Therefore the function

$$
G^{-1}\left(A(z)-A\left(z_{0}\right)+h_{0}(z)\right)
$$

is real analytic in a neighbourhood of the point $z_{0}$ as a composition of real analytic functions. Thus, $f(z)$ is real analytic in a neighbourhood of the point $z_{0}$, too.

Though we get an information on the local properties of solutions to Problem 1, in some cases we can get an information on the global properties, too. Let us illustrate this on a simple example, corresponding to a special choice of the boundary operator $B$.

Corollary 1. Let $F(w)=w^{2}, B f=\frac{\operatorname{Re} f}{|f|^{2}}, a \in \mathcal{C}^{0, \lambda}(\bar{D})$. Then

1) any solution $f$ to Problem 1, which has no zeros in $\bar{D}$, has the following form:

$$
f(z)=\frac{-1}{A(z)+h(z)}
$$


with a function $h \in \mathcal{C}^{1, \lambda}(\bar{D})$, holomorphic in $D$ and satisfying the relations

$$
\begin{gathered}
A(z)+h(z) \neq 0 \text { in } \bar{D} \\
\operatorname{Re}(h(z))=-u_{0}-\operatorname{Re}(A(z)) \text { on } \partial D ;
\end{gathered}
$$

2) if the function a is real analyzic in $D$ then any solution $f$ to Problem 1 , which does not vanish on $\bar{D}$, is real analytic in $D$;

3) if $f$ and $\tilde{f}$ are solutions to Problem 1 , which have no zeros in $\bar{D}$ then we have

$$
\frac{1}{f}-\frac{1}{\tilde{f}}=i c
$$

with a real constant $c$;

4) for each function $u_{0} \in \mathcal{C}^{0, \lambda}(\partial D)$ there is a solution $f$ to Problem 1, which has no zeros in $\bar{D}$.

Proof. If $f$ is a solution to Problem 1 and $f(z) \neq 0$ in $\bar{D}$ then we set

$$
h(z)=\frac{-1}{f(z)}-A(z) .
$$

By the definition the function $h$ is $\mathbb{R}$-differentiable in $D$ and, by direct calculation we get

$$
\bar{\partial} h=\frac{\bar{\partial} f}{f^{2}}-a=0 \text { in } D .
$$

Hence the function $h$ is holomorphic in $D$ and it belongs to $\mathcal{C}^{1, \lambda}(\bar{D})$. In particular, $f$ has the form (1).

Next, the function $f$ is continuous on the compact set $\bar{D}$ and hence it is bounded on $\bar{D}$ by the Weierstrass Theorem. Thus,

$$
\frac{-1}{f(z)}=A(z)+h(z) \neq 0
$$

on $\bar{D}$, i.e. $h$ satisfies relation (2). Moreover, as $\operatorname{Re}(1 / f)=\operatorname{Re}(f) /|f|^{2}$, the function $h$ satisfies also relaton (3). The statement 1 ) is proved.

The statement 2) follows from Theorem 1 immediately.

Let now $f, \tilde{f}$ be solutions to Problem 1 , having no zeros in $\bar{D}$. Then

$$
\frac{1}{f}-\frac{1}{\tilde{f}}=-A(z)-h(z)+A(z)+\tilde{h}(z)=\tilde{h}(z)-h(z),
$$

where $\tilde{h}(z), h(z)$ are holomorphic functions satisfying relation (3). This means that $\tilde{h}(z), h(z)$ are solutions of the Riemann-Hilbert with the same datum $u_{0}$. Therefore $\tilde{h}(z)-h(z)=i c$ with a real constant $c$, see, for instance, [1, Ch. 4, Sec. 29]). The statement 3) is proved.

Finally, as we have seen above, any function of the form (1) is a solution to the equation $\bar{\partial} f=a(z) f^{2}$ in the domain $D$ accept the point where the denominator equals to zero (of course, if the function $h$ is holomorphic in $D$ ). Thus, to solve Problem 1 we need to find a holomorphic function $h(z) \in C^{1, \lambda}(\bar{D})$ in $D$, satisfying relations (2) and (3).

Since $A \in \mathcal{C}^{1, \lambda}(\bar{D})$ and $u_{0} \in \mathcal{C}^{1, \lambda}(\partial D)$, the existence of a holomorphic function $h \in \mathcal{C}^{1, \lambda}(\bar{D})$, satisfying (3) follows from results $[1$, Ch. 4 , Sec. 29]). Moreover, $h$ can be expressed via $A$ and $u_{0}$ by the precise integral formula involving the Green function of the Dirichlet problem for the Laplace operator in $D$. 
As the set $\bar{D}$ is compact and $A(z) \in \mathcal{C}^{1, \lambda}(\bar{D})$, the function is bounded over $\bar{D}$. As we have noted above, any two solutions to Riemann-Hilbert problem with given data differs by a summand $i c$ with a real constant $c$, see, for instannce, [1, Ch. 4, Sec. 29]). Hence, choosing an appropriate real constant $c$ we may guarantee that the correponding function $h$ satisfies (2), too, i.e., that the corresponding function of the type (1) has non zero denominator over the compact $\bar{D}$. Thus, for the particular boundary operator $B$ and each fixed $u_{0} \in \mathcal{C}^{1, \lambda}(\partial D)$ we presented a family of solutions to Problem 1, with elements do not vanishing on $\bar{D}$. This proves the Statement 4).

The work was supported by RFBR grant 14-01-00544 and the grant of President of Russian Federation for leading scientific schools NSh-9149.2016.1.

\title{
References
}

[1] F.D.Gakhov, Boundary Value Problems, Nauka, Moscow, 1977 (in Russian).

[2] E.Wegert, Nonlinear Boundary Value Problems for Holomorphic Functions and Singular Equations, Akademie Verlag, Berlin, 1992.

[3] G.Semmler, E.Wegert, Nonlinear Riemann-Hilbert Problems and Boundary Interpolation, Computational Methods and Function Theory, 3(2003), no. 1, 179-199.

[4] A.I.Shnirel'man, The degree of a quasi-ruled mapping and a nonlinear Hilbert problem, Math. USSR-Sbornik, 18(1972), no. 3, 373-396.

[5] L.V.Wolfersdorf, A Class of Nonlinear Riemann-Hilbert Problems for Holomorphic Functions, Mathematische Nachrichten, 116(1984), no. 1, 89-107.

[6] M.A.Efendiev, W.L.Wendland, Nonlinear Riemann-Hilbert problems for doubly connected domains and closed boundary data. Topological Methods in Nonlinear Analysis, J. of the Juliusz Schauder Center, 17(2001), 111-124.

[7] N.M.Gunther, La théorie du potentiel et ses Applications aux problémes fondamentaux de la physique mathématique, Paris, Gauthier-Villars, 1934.

\section{Об аналоге задачи Римана-Гильберта для одного нелинейного возмущения оператора Коши-Римана}

\author{
Юлия Л. Черепанова \\ Александр А.Шлапунов \\ Институт математики и фундаментальной информатики \\ Сибирский федеральный университет \\ Свободный, 79, Красноярск, 660041
}

Россия

\begin{abstract}
В работе рассмотрено одно нелинейное обобщение хорошо известной задачи Римана- Гильберта о восстановлении голоморфной функции в области по ее вещественной части на границе. Получена информачия о локальной структуре решения для достаточно широкого класса нелинейностей. Указаны достаточные условия вещественной аналитичности решений. Приведен простой пример решения нелинейной задачи.
\end{abstract}

Ключевые слова: оператор Коши-Римана, нелинейная задача Римана-Гильберта 